\title{
Changing features do not guide attention in change detection: Evidence from a spatial cuing paradigm
}

\author{
JENNIFER A. STOLZ \\ University of Waterloo, Waterloo, Ontario, Canada \\ and \\ PIERRE JOLICQEUR \\ Université de Montréal, Montréal, Québec, Canada
}

\begin{abstract}
Smilek, Eastwood, and Merikle (2000) demonstrated that the detection of change was facilitated when the target character changed in many rather than few of its features. Specifically, the function relating search response time to display set size was shallower when more features changed than when fewer features changed. The researchers interpreted these results as indicating that large feature changes provide preattentive guidance of focal attention to the location of the change. We tested this preattentive guidance hypothesis by examining change detection performance in the context of a spatial cuing paradigm. The hypothesis predicts that (1) the cost on invalidly cued trials should be less when more features change than when fewer features change, and (2) the features manipulation should have no effect on validly cued trials. In contrast to these predictions, our results show that cuing effects are equivalent across all levels of feature change and that a robust effect of the features manipulation is observable for both validly and invalidly cued trials. We argue that large feature changes do not provide preattentive guidance and in fact can be detected more readily after attention is already in place at the target location.
\end{abstract}

The ability to detect change across successive visual displays is a skill that is important for human scene processing. As such, change detection has become a popular research topic in cognitive psychology. One important discovery in this literature is that observers experience difficulty detecting change across two successive displays when a blank interval is interspersed between the presentations (e.g., Pashler, 1988; Phillips, 1974; Rensink, O’Regan, \& Clark, 1997; Simons, 2000). This so-called "change blindness" has been attributed to interference of the blank interval with the factors that normally signal change (e.g., Rensink et al., 1997).

Not surprisingly, the degree of change blindness is sometimes modulated by the magnitude of the change (Smilek, Eastwood, \& Merikle, 2000). That is, larger changes produce less change blindness than do smaller changes. As described below, this inverse relation between the degree of change blindness and the magnitude of change has been attributed to large changes producing signals that attract attention to themselves. That is, large feature changes guide attention to the location of the change (Smilek et al., 2000). This paper tests the hypothesis that large change sig-

This work was supported by the National Sciences and Engineering Research Council of Canada. We thank Derek Besner, Evan Risko, Diego Fernandez-Duque, Ron Rensink, and Dan Simons for comments. Send correspondence to J. A. Stolz, Department of Psychology, University of Waterloo, Waterloo, ON, N2L 3G1 Canada (e-mail: jstolz@) watarts.uwaterloo.ca). nals provide preattentive guidance of focal attention to the location of a changing target.

\section{The Role of Features in Change Detection}

Smilek et al. (2000) investigated change blindness in a modified visual search paradigm in which two alternating (nearly) identical displays were separated by a blank interval. One character changed from Display 1 to Display 2, and the participant's task was to detect the location of the changing character. The alternating displays contained characters constructed from combinations of simple horizontal and vertical features. A key manipulation in these experiments was that the degree of change in the target was defined by the number of features that were added or eliminated to construct it. For example, in Experiment 1, the target character changed by either two features (i.e., the character $\mathrm{E}$ changing to an $\mathrm{L}$, or an $\mathrm{L}$ changing to an E) or three features (i.e., the character $\mathrm{F}$ changing to an L, or an L changing to an F). Smilek and colleagues reported that change detection performance was better overall for the three-feature change targets than for the two-feature change targets.

More importantly for the present purposes, Smilek et al.'s (2002) study also manipulated the number of characters in the visual displays. Experiment 1 examined search through set sizes of $4,7,10,13$, or 16 items. The results replicated the well-known set size effect, in which search latencies increase as set size increases (e.g., Wolfe, 1994). Furthermore, the magnitude of the benefit for 
three- versus two-feature change targets increased as the display size increased, a features $\times$ set size interaction.

This features $X$ set size interaction, in which search slopes were shallower when more rather than fewer features changed, was interpreted as evidence that the degree of preattentive guidance of focal attention to the target location varied according to the magnitude of the change. In other words, "attentional guidance based on preattentive comparisons of specific items depends on the size of the discrepancy, whereby larger changes lead to more efficient preattentive guidance than smaller changes." (Smilek et al., 2000, p. 487) Specifically, the ability of large feature changes to guide attention to the target location confers a larger benefit as set size increases, hence the features $\times$ set size interaction.

\section{Do Feature Changes Really Guide Attention?}

The present experiment tests the hypotheses that feature changes provide preattentive guidance and that the degree of preattentive guidance varies according to the magnitude of the change (i.e., the number of features changing). This goal is accomplished by manipulating the number of features changing in a change detection task in conjunction with the manipulation of a second variable whose ability to guide focal attention has been well established - a spatial cue (e.g., Johnston, McCann, \& Remington, 1996; Posner, 1980). Numerous studies have demonstrated that responses to targets are facilitated when the target appears in a location previously occupied by a spatial cue (e.g., Posner, Nissen, \& Ogden, 1978). This spatial cuing effect results from the cue drawing attention to its location. When a target subsequently appears at the cued location on a validly cued trial, attention is present, ready to undertake stimulus processing. In contrast, on an invalidly cued trial, attention will have been misoriented by the cue and will therefore need to be moved to the target location to permit target processing (e.g., Johnston et al., 1996).

There are two predictions that follow from the hypotheses that change signals provide preattentive guidance of focal attention and that the degree of guidance varies in accord with the magnitude of the change. First, the cost of having attention directed to the "wrong," or invalid, location by a cue should be less when more features change than when fewer features change. Because large feature changes guide attention to themselves more readily than do smaller feature changes, a large feature change at an invalidly cued location should provide a stronger signal to reorient attention to the proper location than would a small feature change. This reasoning predicts that the magnitude of the cuing effect (i.e., invalid-valid) should be smaller for large feature change trials than for small feature change trials. Thus, the preattentive guidance hypothesis leads one to predict a spatial cuing $\times$ features changing interaction.

Second, if a large feature change promotes preattentive guidance to the location of the changing target, the features manipulation should not affect performance on valid trials. By definition, attention is present at the target location on a validly cued trial. Therefore, a large feature change should provide no added benefit in summoning attention to the already attended location. This pattern of results also produces a spatial cuing $\times$ features changing interaction.

These two predictions of the preattentive guidance hypothesis were tested by showing observers an initial brief display of three items. A blank interval then intervened, and during this blank interval a cue signaled one of the three potential target locations. The second display then appeared, identical to the first display except at the location of the changed character. The participant's task was to indicate the location of the changed target character. In one third of the trials, the changed target character appeared in the cued location, but in the remaining two thirds of trials the target appeared in one of the uncued locations. The spatial cues were exogenous, or abrupt onset, cues, and because they were not valid predictors of target location (i.e., $33 \%$ valid in a three-location display), observers' responses to the cues should be automatic rather than intentional (e.g., Jonides, 1981). As noted earlier, the number of features defining the target change varied, and the two predictions generated from the preattentive guidance account predict that the joint effects of the two variables, spatial cuing and number of target features changing, should be interactive. The exact nature of the spatial cuing $\times$ features changing interaction is such that cuing effects, based on Prediction 1, should be smaller for greater feature change trials than for smaller feature change trials, and based on Prediction 2, should be characterized by the absence of a features effect on validly cued trials.

\section{METHOD}

\section{Participants}

Thirty-four University of Waterloo undergraduate students participated in Experiment 1 in exchange for \$6. All had normal or correctedto-normal vision.

\section{Design}

Experiment 1 used a 2 (spatial cuing: valid vs. invalid) $\times 3$ (features changing: two vs. three vs. four) within-subjects design. All levels of both variables occurred randomly throughout the experiment.

\section{Stimuli and Visual Displays}

Stimuli were LED-type alphanumeric characters constructed from the combination of basic vertical and horizontal features. Figure 1 displays the eight characters in the stimulus set and the manner in which they were paired in order to create differences of two, three, or four features. For each trial, a level of feature change was determined and an appropriate target change pair was selected without replacement from the stimulus set. Two distractor characters were also selected without replacement to complete the visual display.

The three target characters were drawn on the vertices of an imaginary equilateral triangle that was centered at fixation, and each character subtended a visual angle of approximately $1^{\circ}$ vertically and horizontally at a viewing distance of approximately $63 \mathrm{~cm}$. The triangle was displayed in one of two configurations - with tip pointing up or tip pointing down - and subtended a visual angle of approximately $4.5^{\circ}$. The two triangle configurations were used equally often in all conditions, and the target character appeared in each of the 


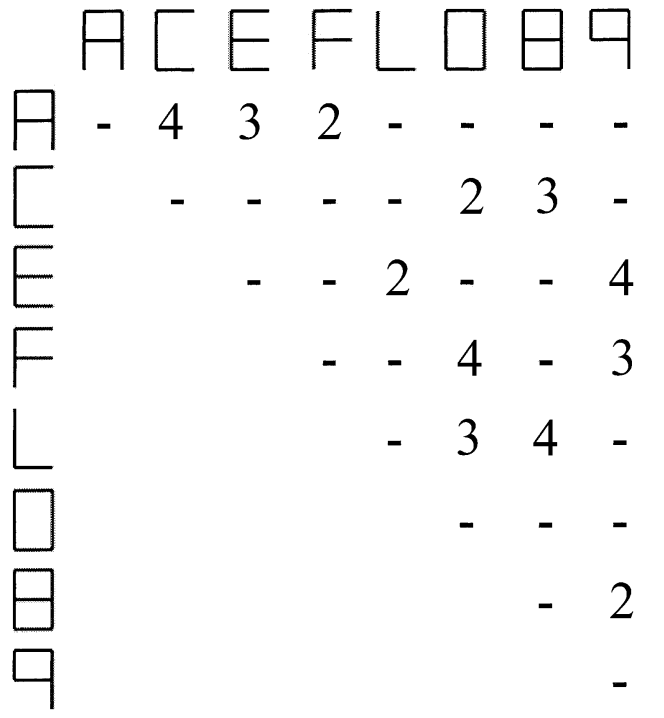

Figure 1. The set of characters used in the experiment. Values contained in the matrix represent the number of features changing for that target character pairing.

three locations equally often in all conditions. All characters appeared as white (MEL code $\mathrm{R}: \mathrm{G}: \mathrm{B}=35: 35: 35$ ) on a black background.

\section{Procedure}

All participants were tested individually in a dimly lit room. Task instructions were displayed visually and supplemented by the experimenter.

Each trial began with a fixation signal $(+)$ that appeared in the center of the screen for $500 \mathrm{msec}$. A 500-msec blank screen followed the disappearance of the fixation signal. The first display of the search characters was exposed for $200 \mathrm{msec}$, oriented as on an imaginary triangle as described above. The disappearance of the first display initiated a blank interval. At $17 \mathrm{msec}$ following the initiation of the blank interval, a spatial cue was displayed for $50 \mathrm{msec}$. The cue was a solid white disc, presented on the radius one pixel past the furthermost point of the item that occupied the cued location in the first display. Following the cue, a second 17-msec blank interval occurred. The second search display was then presented, and remained in view until response. The second display occupied the same locations as did the first display, and two of the three characters were identical to those in the first display. The third, or target character, was new. The change target occupied the location indicated by the spatial cue on in one third of the trials and one of the two uncued locations in the remaining two thirds of the trials.

The task was to identify the location of the changed target character. Participants were instructed to press the left, middle, or right of three adjacent keys on the bottom row of the keyboard to indicate that the target was to the left of fixation, in the center, or to the right of fixation, respectively. A response initiated an intertrial interval of 1,000 msec during which the computer screen was blank. Response time (RT) was measured from the onset of the second visual display. Participants were encouraged to respond as quickly and accurately as possible.

Participants performed six blocks of 128 trials with rest breaks between blocks. Prior to the experiment proper, participants performed one block of 32 practice trials. Practice trials were identical to experimental trials, with the exception that accuracy feedback was given to participants during the practice phase but not in the exper- imental phase. In the practice phase the fixation signal varied to provide accuracy feedback for the prior trial. A "+" sign indicated that performance on the prior trial had been accurate, whereas a "-" sign indicated that an error had been made.

\section{RESULTS}

RT analysis was conducted only for the trials with an accurate search response. These data were subjected to a recursive trimming procedure in which the criterion cutoff for outlier removal was established independently for each participant and each condition by reference to the sample size in that condition (Van Selst \& Jolicœur, 1994). This trimming procedure resulted in the exclusion of less than $2.5 \%$ of the RT data. Following outlier removal, the screened RT data from trials with an accurate response were subjected to a 2 (spatial cuing: valid vs. invalid) $\times 3$ (features changing: two vs. three vs. four) within-subjects ANOVA. A parallel analysis was conducted on the error data.

As illustrated in Figure 2, responses were faster and more accurate following a valid spatial cue $(595 \mathrm{msec}, 6.9 \% \mathrm{er}-$ rors) than following an invalid spatial cue $(672 \mathrm{msec}$, 15.8\% errors): $F(1,31)=26.23, M S_{\mathrm{e}}=10,919.95, p<$ .001 for RTs, and $F(1,31)=32.77, M S_{\mathrm{e}}=115.52, p<$ .001 for errors. The number of features changing at the target location also had a robust effect on performance: $F(2,62)=8.96, M S_{\mathrm{e}}=1,766.01, p<.001$ for RTs, and $F(2,62)=14.79, M S_{\mathrm{e}}=19.40, p<.001$ for errors. Responses had a shorter latency and were more accurate when more features changed than when fewer features changed (i.e., $619 \mathrm{msec}$ and $9.2 \%$ errors for four-feature change targets, $631 \mathrm{msec}$ and $11.3 \%$ errors for three-feature change targets, $650 \mathrm{msec}$ and $13.4 \%$ errors for two-feature change targets), replicating the effects of Smilek et al. (2000).

Clearly, the present experiment produced robust effects from both our spatial cuing and features changing manipulations. This result indicates that the spatial cues were effective in guiding attention and that the features manipulation affected search efficiency. The central question is whether these factors had interactive effects on RTs as predicted by the preattentive guidance hypothesis. Most notably, if a smaller cost was associated with invalidly cuing a four- versus a two- or three-feature change target, this result would be consistent with the hypothesis that larger numbers of changing features give off stronger signals than do smaller numbers of changing features and guide focal attention to the location of the change more effectively. Furthermore, if the features variable did not affect performance on validly cued trials, this would also be consistent with the preattentive guidance hypothesis. In contrast, a failure to find these interactive effects would indicate strong evidence against the preattentive guidance hypothesis. As both Figure 2 and the results of the analysis clearly show, the effects of the spatial cuing and feature change variables were additive on response times $(F<1)$. If anything, the cuing effect was slightly larger for four- 


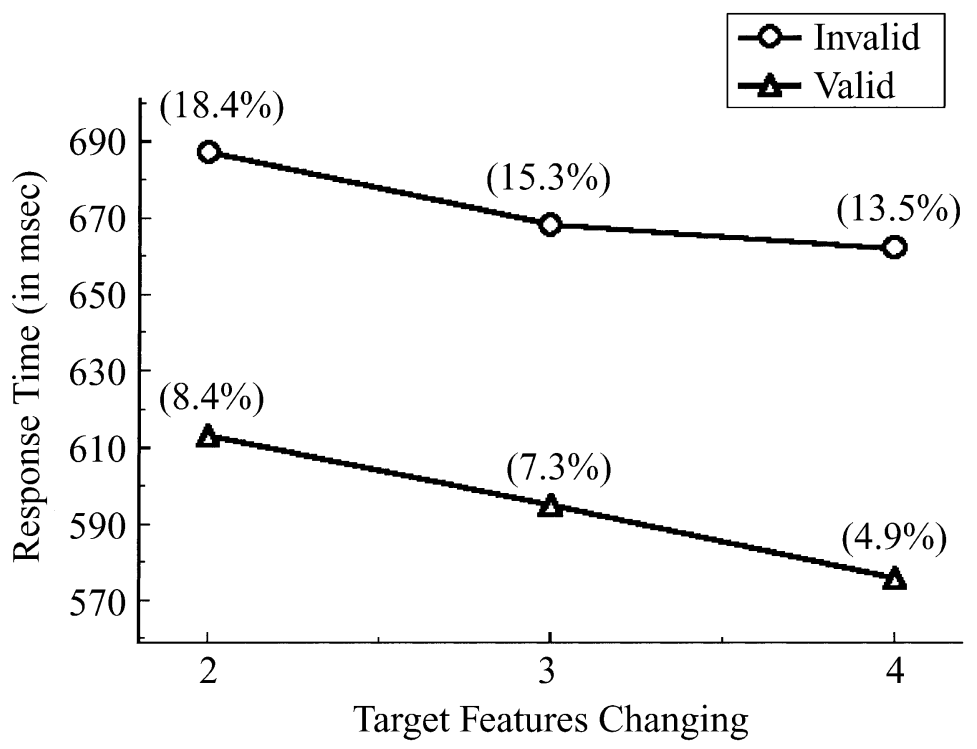

Figure 2. Mean response times (in milliseconds) and percent errors for validly and invalidly cued trials at each level of features changing. The $95 \%$ confidence interval surrounding each response time is \pm 13 msec (Loftus \& Masson, 1994).

than for two- or three-feature change targets, and the magnitude of the features effect tended to be larger (fourfeature-two-feature difference) for validly cued trials $(37 \mathrm{msec})$ than for invalidly cued trials $(25 \mathrm{msec})$. The absence of an interaction in the error data is also noted $(F<1)$.

The claim that cuing effects were equivalent for each level of feature change is central to our conclusions. We therefore conducted power analyses to demonstrate that our experiment had adequate power to detect the spatial cuing $\times$ features changing interaction. The power analyses tested the strength of our experiment to evaluate two hypotheses. The strong hypothesis was that the invalidvalid cuing effect would be eliminated in the four-feature change condition. Using the method described by Cohen (1988, pp. 48-52) for determining the power to detect differences between paired observations in a within-subjects manipulation such as ours, we estimated our power to detect the elimination of the cuing effect for the four-feature change targets. Our analysis indicated that such a reduction would constitute a large effect in Cohen's terms $(d>$ 1.9 in this case), and in fact our power to detect such a reduction exceeded 99\%. A second power analysis tested the weaker hypothesis that the cuing effect would not be eliminated in the four-feature change condition, but that it would be reduced to approximately half of the 73-74 msec effect seen for two- and three-feature change targets. We conducted this analysis to counter the charge that our spatial cuing manipulation was so powerful that it could not be entirely eliminated, and therefore sought to test our power to demonstrate a modest reduction in the magni- tude of the cuing effect. Importantly, our power to detect even this 37-msec reduction in the cuing effect exceeded $99 \%$, owing to the facts that (1) the variability associated with cuing effects was so small and (2) the cuing effects at each level of feature change were highly correlated, $r \mathrm{~s} \approx .74$. Both of these factors contributed to an effect size $d$ that approached 1 . We are therefore confident that this experiment had extremely high power to detect a spatial cuing $\times$ features changing interaction if it were present.

\section{DISCUSSION}

The present experiment tested the hypotheses that changing features provide preattentive signals to summon focal attention to the location of a changing target and that the degree of strength held by a preattentive signal is directly linked to the number of features changing. It tested these ideas by examining the effects of a target feature manipulation in the context of a spatial cuing manipulation. Spatial cuing manipulations using exogenous cues, such as the one presented here, have been argued to affect input attention (e.g., Johnston et al., 1996). That is, they act as a gatekeeper, moving input attention to the location of an event or object so that processing on the object may begin. The key question here was whether the changing features themselves also act to direct focal attention.

If changing features provide a preattentive signal, thereby summoning focal attention to the location of the change, then two specific predictions follow. First, the cost of cuing attention to the wrong location by use of an invalid cue will be less for large feature change trials than for 
small feature change trials. That is, the potent preattentive signal emanating from a change in many features will quickly orient focal attention to the proper location, lessening the impact of the invalid cue. In stark contrast to this prediction, we found that the effects of the cuing manipulation were not modulated by the number of features changing at the target location. Instead, large and consistent spatial cuing effects were seen at each level of feature change.

Second, if changing features provide a preattentive signal, there should be no need for this signal on a validly cued trial, as attention is already at the target location when the target appears. According to this analysis, the effect of the features manipulation should be nil on a validly cued trial. Once again, in stark contrast to the predictions from the preattentive guidance account, the features changing variable had a robust effect on performance, and this effect was consistent for both validly and invalidly cued trials.

These results join a growing body of work that challenges the view that change detection can be accomplished in the absence of attention (e.g., Mitroff \& Simons, 2002; Mitroff, Simons, \& Franconeri, 2002). For instance, Mitroff et al. demonstrated that the original features changing $X$ set size interaction reported by Smilek et al. (2000) could not be accounted for by the implicit accumulation over a number of displays of change location information. In Mitroff's Experiment 3, the researchers changed the location of all characters on each successive cycle (one cycle $=$ display $\mathrm{A}+$ change display $\mathrm{A}_{1}$ ). Despite the fact that the display characters changed screen position on each successive cycle, thereby eliminating the possibility that implicit change location information was building up across displays, Mitroff's team nonetheless replicated the features changing $\times$ set size interaction. While their location change manipulation and the associated results left open the possibility that implicit detection guided attention within a single cycle of displays, the present results clearly refute that account. Moreover, Fernandez-Duque and Thornton (2000; see also Thornton \& Fernandez-Duque, 2000) demonstrated via a postcuing paradigm that there is little evidence that attention is cued or implicitly drawn to the location of a change on a trial in which no explicit detection of the change has been registered. Yet, despite the fact that little or no evidence indicates that changing features provide preattentive guidance of attention, the features changing variable had robust effects on performance in the present experiment. How, then, do changing features affect change detection performance?

We argue that the present results, demonstrating that the spatial cuing effect is not modulated by the number of features changing at the target location, are most consistent with the view that attentional guidance and features changing have independent effects on change detection performance. Specifically, the effects of spatial cuing and features changing on search performance are best explained by proposing that the number of features changing exerts its effect on performance after attention has been summoned to the location of the target, either by a valid cue or a reorientation of attention to one of the uncued locations following an invalid cue. In the present paradigm, for example, attention is oriented to a location following the appearance of a spatial cue. The second display appears shortly thereafter, and the participant determines whether the location holding attention contains the target character. Large magnitude changes (i.e., many features changing) are detected with a higher probability, and with a shorter latency, even on validly cued trials. If the cued location does not hold the target, attention is reoriented to one of the remaining locations, and the search begins anew. Search in these uncued locations is also affected by the number of features changing: Larger changes are detected with higher probabilities and shorter latencies.

This same serial-processing argument provides an excellent account of the original features changing $\times$ set size interaction demonstrated by Smilek et al. (2000) and replicated by Mitroff et al. (2002). One major difference between the present experiment and the two previous studies is that in our experiment the orientation of attention was controlled by the onset of the exogenous cue, whereas the previous experiments had allowed the participant to determine the movement of attention throughout the visual display. After thus controlling attention, we argue that the search process and the effects of the features changing manipulation on it were the same in both the controlled and uncontrolled paradigms. That is, greater feature changes produce a greater likelihood of target detection during the serial search process, thereby obviating the need to posit preattentive guidance of attention via implicit processes.

\section{SUMMARY AND CONCLUSIONS}

The results of the present experiment investigating the joint manipulation of spatial cuing and the number of features changing at the target location are clear. The hypothesis that large feature changes benefit performance by providing a preattentive guidance signal lends itself to the predictions that the effects of cuing should be less for large than for small feature changes and that the effects of features changing should be nil on validly cued trials. In stark contrast to these predictions, the effects of spatial cuing and features changing were robust and independent of one another. This pattern of results is most consistent with the views that spatial cuing guides attention to locations in the display and that the benefit of large feature changes arises after the effect of attentional guidance, during the search and comparison processes.

\section{REFERENCES}

CoHEN, J. (1988). Statistical power analysis for the behavioral sciences (2nd ed.). Hillsdale, NJ: Erlbaum.

Fernandez-Duque, D., \& Thornton, I. M. (2000). Change detection without awareness: Do explicit reports underestimate the representation of change in the visual system? Visual Cognition, 7, 323-344.

Johnston, J. C., McCann, R. S., \& Remington, R. W. (1996). Selective attention operates at two processing loci. In A. F. Kramer, M. G. H. Coles, \& G. D. Logan (Eds.), Essays in honor of Charles Eriksen (pp. 439-458). Hillsdale, NJ: Erlbaum.

JoNIDES, J. (1981). Voluntary vs. automatic control over the mind's eye's 
movement. In J. Long \& A. D. Baddeley (Eds.), Attention and performance IX (pp. 187-204). Hillsdale, NJ: Erlbaum.

Loftus, G. R., \& MAsson, M. E. J. (1994). Using confidence intervals in within-subject designs. Psychonomic Bulletin \& Review, 1, 476-490.

Mitroff, S. R., \& Simons, D. J. (2002). Changes are not localized before they are explicitly detected. Visual Cognition, 9, 937-968.

Mitroff, S. R., Simons, D. J., \& Franconeri, S. L. (2002). The siren song of implicit change detection. Journal of Experimental Psychology: Human Perception \& Performance, 28, 798-815.

PASHLER, H. (1988). Familiarity and visual change detection. Perception \& Psychophysics, 44, 369-378.

PhILliPs, W. A. (1974). On the distinction between sensory storage and short-term visual memory. Perception \& Psychophysics, 16, 283-290.

Posner, M. I. (1980). Orienting of attention. Quarterly Journal of Experimental Psychology, 32, 3-25.

Posner, M. I., Nissen, M. J., \& OgDen, W. C. (1978). Attended and unattended processing nodes: The role of set for spatial locations. In
H. L. Pick \& B. J. Saltzman (Eds.), Modes of perceiving and processing information (pp. 137-157). Hillsdale, NJ: Erlbaum.

Rensink, R. A., O'Regan, J. K., \& Clark, J. J. (1997). To see or not to see: The need for attention to perceive changes in scenes. Psychological Science, 8, 368-373.

SimONS, D. J. (2000). Current approaches to change blindness. Visual Cognition, 7, 1-16.

SmileK, D., EAstwood, J. D., \& Merikle, P. M. (2000). Does unattended information facilitate change detection? Journal of Experimental Psychology: Human Perception \& Performance, 26, 480-487.

Thornton, I. M., \& Fernandez-DuQue, D. (2000). An implicit measure of undetected change. Spatial Vision, 14, 21-44.

VAN SElst, M., \& Jolicceur, P. (1994). A solution to the effect of sample size on outlier elimination. Quarterly Journal of Experimental Psychology, 47A, 631-650.

Wolfe, J. M. (1994). Guided Search 2.0: A revised model of visual search. Psychonomic Bulletin \& Review, 1, 202-238.

(Manuscript received April 10, 2003;

revision accepted for publication October 15, 2003.) 